ARTIGO ORIGINAL

\title{
Avaliação dos efeitos do adesivo, gramatura e pressão na qualidade de painéis EGP de Eucalyptus badjensis
}

\author{
Evaluation of effects of adhesive, grammage and pressure on the quality of Edge \\ EGP of Eucalyptus badjensis \\ Setsuo Iwakiri' (D), Rosilani Trianoski ${ }^{1}$ (D), Renan Raia Zunta ${ }^{1}$ (D), Giuliano Ferreira Pereira ${ }^{1}$ (), \\ Thiago Souza Rosa ${ }^{1}$ (D) \\ ${ }^{1}$ Universidade Federal do Paraná - UFPR. Curitiba, PR, Brasil
}

Como citar: Iwakiri, S., Trianoski, R., Zunta, R. R., Pereira, G. F., Rosa, T. S. (2021). Avaliação dos efeitos do adesivo, gramatura e pressão na qualidade de painéis EGP de Eucalyptus badjensis. Scientia Forestalis, 49(129), e3437. https://doi.org/10.18671/scifor.v49n129.20

\begin{abstract}
Resumo
O objetivo deste trabalho foi avaliar o potencial do uso da madeira de Eucalyptus badjensis para produção de painéis EGP, e os efeitos do tipo de adesivo, gramatura e pressão na resistência da colagem. Foram avaliadas as propriedades físicas da madeira e a resistência das emendas de topo e da colagem lateral, utilizando adesivo PVA e EPI, nas gramaturas de 160, 180 e $200 \mathrm{~g} / \mathrm{m}^{2}$, e pressão específica de 0,50, 0,75 e $1,0 \mathrm{MPa}$. Os resultados indicaram que a madeira de $E$. badjensis apresenta média massa específica básica $\left(0,475 \mathrm{~g} . \mathrm{cm}^{-3}\right)$ e alto fator de anisotropia de contração $(2,35)$. As juntas coladas com adesivo EPI, com maiores gramaturas $\left(200 \mathrm{~g} / \mathrm{m}^{2}\right)$ e pressão $(1,0 \mathrm{MPa})$ apresentaram melhores resultados de cisalhamento da linha de cola. Com base nos requisitos das normas ASTM D 5572 para emendas de topo e a norma EN 15353-2008 ( $5^{\circ}$ percentil inferior) para a colagem lateral, pode-se afirmar que a madeira de Eucalyptus badjensis apresenta potencial para produção de painéis EGP com adesivo EPI, visando aplicações em ambiente úmido.
\end{abstract}

Palavras-chave: Acetato de polivinila; Emulsão polimérica de isocianato, Cisalhamento da linha de cola.

\section{Abstract}

The objective of this work was to evaluate the potential use of Eucayptus badjensis wood for the production of edge glued panels - EGP. The physical properties of the wood, finger joint and edge gluing, using PVA and EPI adhesive in the grammage of 160,180 and $200 \mathrm{~g} / \mathrm{m}^{2}$ and specific pressure of $0.50,0.75$ and $1.0 \mathrm{MPa}$ were evaluated. The results indicated that $E$. badjensis wood presents medium basic density $\left(0,475 \mathrm{~g} \cdot \mathrm{cm}^{-3}\right)$ and high contraction anisotropy $(2,35)$. The edge-glued joints with EPI adhesive, with greater grammage $\left(200 \mathrm{~g} / \mathrm{m}^{2}\right)$ and pressure $(1.0 \mathrm{MPa})$, presented better results of glue line shear strength. Based on the requirements of ASTM D 5572 standards for finger joints and the EN 15353-2008 (5th percentile lower) for edge gluing, it can be stated that Eucalyptus badjensis wood has great potential for EGP production with EPI adhesive, with applications in humid environments.

Keywords: Polyvinyl acetate; Polymeric emulsion of isocyanate; Glue line shear test.

\section{INTRODUÇÃO}

A utilização da madeira serrada pode apresentar várias limitações como dimensões, anisotropia e defeitos naturais que afetam as suas propriedades físicas e mecânicas. Entretanto, por meio da colagem de madeiras com emprego de adesivos é possível obter produtos reconstituídos de madeira, com maiores dimensões, melhor estabilidade

Fonte de financiamento: nada a declarar

Conflito de interesse: nada a declarar

Autor correspondente: setsuo.ufpr@gmail.com

Recebido: 31 julho 2019

Aceito: 02 abril 2020.

Editor: Paulo Henrique Müller Silva.

(c) (i) Este é um artigo publicado em acesso aberto (Open Access) sob a licença Creative Commons Attribution, que permite uso, distribuição e c) reprodução em qualquer meio, sem restrições desde que o trabalho original seja corretamente citado. 
dimensional e distribuição da resistência mecânica, com ganhos em qualidade e relação custo-benefício (Iwakiri, 2005).

Dentre os produtos reconstituídos de madeira, encontram-se os painéis colados lateralmente (EGP), os quais são compostos por sarrafos unidos por meio de colagem lateral, podendo ou não ser unidos por emendas de topo "finger joints" (Tienne et al., 2011). Os principais tipos de adesivos empregados na produção de painéis EGP são o poliacetato de vinila (PVA) e emulsão polimérica de isocianato (EPI).

O PVA é um adesivo resultante da polimerização do acetato de vinila, sendo popularmente conhecido como "cola branca". O produto colado com PVA apresenta alta resistência mecânica em ambiente seco, porém limitações de uso em ambientes com altas temperaturas e umidade relativa (Pizzi, 1983). Já, o adesivo EPI é composto por uma base de poliacetato de vinila e um isocianato polimérico, cuja função é catalisar a cura do adesivo, que ocorre por meio da reação dos grupos isocianatos com os grupos hidroxilas da madeira. Possui alta resistência à água e temperatura, boa estabilidade, cura rápida, porém apresenta como desvantagem seu maior custo (Frihart \& Hunt, 2010).

A colagem de duas peças de madeira depende de vários fatores como a sua estrutura anatômica, massa específica e porosidade. Iwakiri (2005) relata que, as propriedades anatômicas da madeira estão relacionadas diretamente com as ações de mobilidade do adesivo na formação de "ganchos" de ligação entre as faces de colagem. A porosidade tem uma relação inversa com a massa específica, ou seja, madeira com alta massa específica possui menos espaços vazios e, consequentemente, dificulta a penetração do adesivo, resultando na baixa ancoragem e adesão.

A quantidade de adesivo (gramatura) na formação da linha de cola entre duas peças de madeira é um dos parâmetros importante para promover uma boa colagem. De acordo com Fonte \& Trianoski (2015), quando a gramatura é menor ou maior que a ideal, a resistência do produto colado pode ser alterada. Gramaturas muito baixas implicam em resistência inferior devido à aderência e ancoragem insuficientes. Já com gramaturas elevadas, pode ocorrer vazamento do adesivo em excesso pelas bordas dos painéis, resultando em prejuízos econômicos (Marra, 1992). Iwakiri et al. (2014) relatam que a faixa de gramaturas mais empregadas pelas indústrias varia de 180 a $220 \mathrm{~g} / \mathrm{m}^{2}$ para o PVA e de 150 a $300 \mathrm{~g} / \mathrm{m}^{2}$ para o EPI.

A pressão aplicada durante a prensagem da madeira tem a função de promover o contato entre o adesivo e a madeira, promovendo a penetração do mesmo nos poros do substrato, com a formação de uma película adesiva fina, uniforme e com a resistência necessária para manter a união de duas peças coladas (Lopes, 2008; Frihart \& Hunt, 2010). A pressão aplicada varia de acordo com a massa específica da madeira, sendo que madeiras de alta massa específica requerem pressões mais altas, em comparação com as madeiras de baixa massa específica (Marra, 1992; Tienne, 2006). Fabricantes de adesivos recomendam pressão específica de 0,6 a 1,0 MPa para colagem lateral da madeira com PVA, e de 0,8 a 1,2 MPa para EPI, dependendo da sua massa específica (Akzo Nobel Perú S. A. C., 2010).

Para emendas de topo do tipo finger joint, a pressão a ser aplicada depende do comprimento dos dentes e da massa específica da madeira, sem exceder o limite máximo para que não ocasione fissuras com uma extensão superior a $0,5 \mathrm{~cm}$ no fundo dos dentes (Serrano, 2009).

As principais espécies empregadas comercialmente na produção de painéis EGP no Brasil são o Pinus taeda, Eucalyptus grandis e Tectona grandis. Várias espécies têm sido estudadas quanto à sua viabilidade para produção de EGP, sendo que as espécies de eucalipto apresentam alto potencial tendo em vista seu rápido crescimento. Entretanto, a maioria dessas espécies apresenta madeira com baixa estabilidade dimensional, sendo necessário uso de recursos tecnológicos para seu aproveitamento, como por meio de colagem de pequenas peças para formação de produtos com maiores dimensões e melhor estabilidade dimensional, tais como os painéis EGP.

O Eucalyptus badjensis Beuzev. \& Welch é uma espécie nativa das regiões montanhosas da Austrália. Por apresentar elevadas taxas de crescimento e resistência à geada, a espécie apresenta grande potencial para plantios em regiões frias do Brasil (Higa et al., 2002). A 
utilização de Eucalyptus badjensis vem sendo estudada para os mais variados produtos de origem florestal, como óleos essenciais, celulose e papel (Mantero et al., 2008). Contudo, estudos relacionados à sua utilização para produção de painéis de madeira reconstituídos são ainda incipientes.

Perante o exposto, este trabalho teve como objetivo avaliar os efeitos do tipo de adesivo, gramatura e pressão específica na colagem de madeira de Eucalyptus badjensis para produção de painéis de colagem lateral - EGP.

\section{MATERIAL E MÉTODOS}

Foram utilizadas nesta pesquisa, madeiras de Eucalyptus badjensis, com 13 anos de idade, proveniente de povoamentos florestais localizados no município de Lajes - SC. Foram coletadas três árvores, as quais foram seccionadas em toras com 2,0 m de comprimento e desdobradas em tábuas com $25 \mathrm{~mm}$ de espessura. Após secagem em estufa convencional até o teor de umidade médio de $12 \%$, as tábuas foram serradas e aplainadas em sarrafos com dimensões finais de $22 \mathrm{~mm}$ (espessura) $\times 60 \mathrm{~mm}$ (largura) $\times 420 \mathrm{~mm}$ (comprimento) para colagem lateral, e $22 \mathrm{~mm} \times 60 \mathrm{~mm} \times 210 \mathrm{~mm}$ para colagem de topo. Estes passaram por processo de usinagem de topo do tipo "finger joints".

Das tábuas centrais, das posições radiais, foram retirados os corpos-de-prova para determinação de propriedades físicas (massa específica básica, massa específica aparente, contração volumétrica e anisotropia de contração), com base nos procedimentos descritos na norma COPANT 461 (Comissão Panamericana de Normas Técnicas, 1972b e 459 (Comissão Panamericana de Normas Técnicas,1972a).

Para a colagem de topo, foi utilizado o adesivo poliacetato de vinila - PVA D3, aplicado em gramatura de $180 \mathrm{~g} / \mathrm{m}^{2}$, pressão de $3 \mathrm{MPa}$ e tempo de prensagem de 10 segundos. Para a colagem lateral, foram utilizados os adesivos PVA e EPI, com gramaturas de 160, 180 e $200 \mathrm{~g} / \mathrm{m}^{2}$, pressão específica de 0,50, 0,75 e 1,0 MPa, por um tempo de 2 horas. O plano experimental está apresentado na Tabela 1.

Tabela 1. Delineamento experimental (adesivo, gramatura e pressão) utilizados na pesquisa

\begin{tabular}{cccc}
\hline Tratamento & Adesivo & Gramatura $\left(\mathbf{g} / \mathbf{m}^{2}\right)$ & Pressão $(\mathbf{M P a})$ \\
\hline 1 (PVA-160/0,5) & PVA & 160 & 0,50 \\
2 (EPI-160/0,5) & EPI & 160 & 0,50 \\
$3($ PVA-180/0,5) & PVA & 180 & 0,50 \\
$4($ EPI-180/0,5) & EPI & 180 & 0,50 \\
$5($ PVA-200/0,5) & PVA & 200 & 0,50 \\
$6($ EPI-200/0,5) & EPI & 200 & 0,50 \\
$7($ PVA-160/0,75) & PVA & 160 & 0,75 \\
$8($ EPI-160/0,75) & EPI & 160 & 0,75 \\
$9($ PVA-180/0,75) & PVA & 180 & 0,75 \\
$10($ EPI-180/0,75) & EPI & 180 & 0,75 \\
$11(P V A-200 / 0,75)$ & PVA & 200 & 0,75 \\
$12($ EPI-200/0,75) & EPI & 200 & 0,75 \\
$13($ PVA-160/1,0) & PVA & 160 & 1,0 \\
$14($ EPI-160/1,0) & EPI & 160 & 1,0 \\
$15(P V A-180 / 1,0)$ & PVA & 180 & 1,0 \\
$16(E P I-180 / 1,0)$ & EPI & 180 & 1,0 \\
$17($ PVA-200/1,0) & PVA & 200 & 1,0 \\
$18(E P I-200 / 1,0)$ & EPI & 200 & 1,0 \\
\hline
\end{tabular}

EPI: Emulsão Polimérica de Isocianato; PVA: Poliacetato de vinila. 
Após a estabilização e cura total do adesivo, as juntas coladas (lateral e topo) foram seccionadas para confecção de corpos de prova conforme os procedimentos descritos nas normas ASTM D 5572(American Society for Testing and Materials, 2005) e EN 13354 (European Committee for Standardization, 2008b).

Para a colagem de topo foram testados 30 corpos de prova para cada pré-tratamento: seco (acondicionados à temperatura de $20 \pm 3^{\circ} \mathrm{C}$ e umidade relativa de $65 \pm 5 \%$ ), alta temperatura (exposição por 6 horas à temperatura de $104+3^{\circ} \mathrm{C}$ ) e ciclo triplo $(4$ horas de imersão em água à temperatura de 19 a $27^{\circ} \mathrm{C}$, seguido de secagem à temperatura de $41 \pm 3^{\circ} \mathrm{C}$ por 19 horas; repete-se o procedimento por 3 ciclos). Para a colagem lateral foram testados 15 corpos de prova para cada tratamento e pré-tratamentos: seco (acondicionados à temperatura de $20 \pm 3^{\circ} \mathrm{C}$ e umidade relativa de $65 \pm 5 \%$ ) e úmido ( 24 horas de imersão em água fria à temperatura de $20 \pm 3^{\circ} \mathrm{C}$ ).

Os resultados foram confrontados com os requisitos mínimos estabelecidos na norma ASTM D 5572 (American Society for Testing and Materials, 2005), e EN 13353 (European Committee for Standardization, 2008a). Após os ensaios de cisalhamento, foram avaliadas as percentagens de falhas na madeira na superfície de ruptura da linha de cola.

O delineamento estatístico utilizado foi o inteiramente casualizado, com arranjo fatorial de $2 \times 2 \times 3$, para dois adesivos, três gramaturas e três níveis de pressão. Os dados foram avaliados por meio de teste de outliers, teste de Bartlett para homogeneidade das variâncias, ANOVA em arranjo fatorial e comparação de médias pelo teste de Tukey ao nível de probabilidade de $95 \%$. Os testes estatísticos foram realizados a partir do pacote estatístico Statgraphics XVII.

\section{RESULTADOS E DISCUSSÕES}

\section{Propriedades físicas da madeira de Eucalyptus badjensis}

Na Tabela 2 estão apresentados os resultados médios de massa específica básica, massa específica aparente, contração volumétrica e anisotropia de contração, obtidos para a madeira de Eucalyptus badjensis.

Tabela 2. Resultados médios das propriedades físicas da madeira

\begin{tabular}{ccc}
\hline Propriedade & Valor médio & Coeficiente de Variação (\%) \\
\hline Massa especifica básica $\left(\mathrm{g} \cdot \mathrm{cm}^{-3}\right)$ & 0,475 & 6,59 \\
Massa especifica aparente $\left(\mathrm{g} \cdot \mathrm{cm}^{-3}\right)$ & 0,585 & 7,28 \\
Contração volumétrica (\%) & 16,11 & 22,66 \\
Anisotropia de contração & 2,35 & 21,31 \\
\hline
\end{tabular}

Os valores médios de massa específica básica e aparente da madeira de $E$. badjensis foram respectivamente de $0,475 \mathrm{~g} / \mathrm{cm}^{3}$ e $0,585 \mathrm{~g} / \mathrm{cm}^{3}$, sendo classificada como de média massa específica básica, segundo Arostegui (1982), cujos valores devem se situar no intervalo de 0,410 a $0,600 \mathrm{~g} / \mathrm{cm}^{3}$. A massa específica básica obtida para $E$. badjensis está próxima dos valores mencionados por Lorenzi (2002) para algumas espécies de eucaliptos como $E$. grandis $\left(0,478 \mathrm{~g} / \mathrm{cm}^{3}\right)$, E. urograndis $\left(0,502 \mathrm{~g} / \mathrm{cm}^{3}\right)$ e E. saligna $\left(0,462 \mathrm{~g} / \mathrm{cm}^{3}\right)$.

Cabe ressaltar ainda que, as espécies mais empregadas na produção de painéis EGP no Brasil são de baixa e média massa específica, devido à maior porosidade da madeira e facilidade para penetração de adesivos de alta viscosidade, como as espécies apresentadas a seguir: Tectona grandis $\left(0,480\right.$ a $\left.0,640 \mathrm{~g} / \mathrm{cm}^{3}\right)$, Pinus elliottii e Pinus taeda $\left(0,320\right.$ a 0,340 g/ $\left./ \mathrm{cm}^{3}\right)$ e E. grandis $\left(0,390\right.$ a $\left.0,501 \mathrm{~g} / \mathrm{cm}^{3}\right)$ (Lobão et al., 2011).

O valor médio de contração volumétrica foi de $16,11 \%$, o qual situa se dentro da faixa de $10,2 \%$ a 19,2\% mencionado por Tsoumis (1991) para madeiras de folhosas. 
O valor médio de anisotropia de contração da madeira de $E$. badjensis foi de 2,35, sendo considerada de média a alta anisotropia, segundo a classificação citada por Klitzke (2007), com valores na faixa de 2,1 a 2,5 .

De maneira geral, altos valores de variações dimensionais e anisotropia de contração são características indesejáveis à madeira, limitando o seu uso para diversas finalidades e exigindo técnicas adequadas de processamento e utilização (Keinert Junior et al., 1992). Entretanto, por meio de colagem de peças de madeira serradas obtidas e distribuídas aleatoriamente, como no caso de painéis EGP, pode se minimizar os efeitos negativos da alta anisotropia de determinadas espécies.

\section{Resultados médios de resistência das emendas de topo - Finger Joints.}

Na Tabela 3 estão apresentados os resultados médios de flexão estática e tração paralela das emendas de topo, além de percentagens de falhas na madeira, após os pré-tratamentos a seco, alta temperatura e ciclo triplo.

Tabela 3. Resultados médios de resistência das emendas de topo

\begin{tabular}{cccc}
\hline \multirow{2}{*}{ Pré-tratamentos } & Flexão estática (MPa) & \multicolumn{2}{c}{ Tração } \\
\cline { 3 - 4 } & & Resistência (MPa) & FM (\%) \\
\hline Seco & $48,89(6,85)$ & $26,78(19,39)$ & 97 \\
Alta temperatura & $41,31(13,71)$ & $24,85(30,21)$ & 38 \\
Ciclo triplo & $11,44(50,28)$ & $19,88(50,13)$ & 25 \\
\hline
\end{tabular}

FM: falhas na madeira; valores entre parenteses indicam os coeficientes de variação em percentual.

Os valores médios de resistência à flexão estática das emendas de topo foram de 48,89 MPa, 41,31 MPa e 11,44 MPa, respectivamente para os ensaios a seco, alta temperatura e ciclo triplo. Os valores obtidos para os ensaios de flexão estática após pré-tratamentos a seco e ciclo triplo atenderam aos requisitos da norma ASTM D 5572 (American Society for Testing and Materials, 2005), cujos valores mínimos são respectivamente de 13,8 e 6,9 MPa.

Com relação a alguns resultados apresentados na literatura, Juízo (2015) encontraram para madeiras de Eucalyptus grandis e Eucalyptus saligna, valores médios de flexão estática das emendas de topo de $62,64 \mathrm{MPa}$ e $28,76 \mathrm{MPa}$, respectivamente para ensaios a seco e ciclo triplo. Almeida (2015) encontrou para madeira de Cupressus lusitânica, valores médios de 15,43 MPa e 11,41 MPa; Fonte (2016) encontrou para madeira de Cryptomeria japônica, valores médios de 41,00 MPa e 25,40 MPa; Lau (2017), encontrou para madeira de Populus deltoides, valores médios de $28,80 \mathrm{MPa}$ e 5,49 $\mathrm{MPa}$, respectivamente, para pré-tratamentos a seco e ciclo triplo.

Como pode se constatar, existem grandes variações entre os resultados apresentados pelos referidos autores. Vick (1999) e Prata (2010) afirmam que as variações na massa específica e anisotropia de contração da madeira de diferentes espécies influenciam na resistência das emendas de topo do tipo finger joint, apresentando uma relação direta entre estas variáveis.

Os valores médios de tração paralela das emendas de topo foram respectivamente de $26,78 \mathrm{MPa}, 24,85 \mathrm{MPa}$ e $19,88 \mathrm{MPa}$, para os ensaios a seco, alta temperatura e ciclo triplo. Os resultados atenderam aos requisitos da norma ASTM D 5572 (American Society for Testing and Materials, 2005), cujos valores mínimos são respectivamente de 13,80 MPa e 6,90 MPa para os pré-tratamentos a seco e ciclo triplo.

Com relação à percentagem de falhas na madeira, os valores médios de $97 \%$ e $25 \%$, obtidos para pré-tratamentos a seco e ciclo triplo, atenderam aos requisitos mínimos da norma ASTM D 5572 (American Society for Testing and Materials, 2005), de 30\% e 15\%, respectivamente, para os dois pré-tratamentos avaliados. 
Os resultados obtidos para ensaios de tração paralela das emendas de topo estão compatíveis com os apresentados na literatura para algumas espécies estudadas. Almeida (2015) encontrou para madeira de Cupressus lusitânica, valores médios de 9,37 MPa e 4,04 MPa; Fonte (2016) encontrou para madeira de Cryptomeria japônica, valores médios de 21,30 MPa e 24,40 MPa; Lau (2017) encontrou para madeira de Populus deltoides, valores médios de 24,91 MPa e 6,18 MPa, respectivamente, para pré-tratamentos a seco e ciclo triplo; Juízo (2015) obteve para mistura de madeiras de Eucalyptus grandis e Eucalyptus saligna, valores de flexão estática de $62,64 \mathrm{MPa}, 44,07 \mathrm{MPa}$ e $28,75 \mathrm{MPa}$, respectivamente para os prétratamentos a seco, alta temperatura e três ciclos; e, valores de tração perpendicular de 35,25 MPa, 32,25 MPa e 24,70 MPa, respectivamente para os três pré-tratamentos.

\section{Resultados médios de cisalhamento das juntas coladas.}

$\mathrm{Na}$ Tabela 4 estão apresentados os resultados médios dos ensaios de cisalhamento das juntas coladas para os tratamentos avaliados, após os pré-tratamentos a seco e úmido.

Tabela 4. Resultados médios de resistência da linha de cola ao cisalhamento

\begin{tabular}{|c|c|c|c|c|c|c|}
\hline \multirow{2}{*}{ Tratamento } & \multicolumn{3}{|c|}{ Seco (Curado) } & \multicolumn{3}{|c|}{ 24h Água fria (Úmido) } \\
\hline & RLC (MPa) & $5^{\circ} \mathrm{PI}(\mathrm{MPa})$ & FM (\%) & RLC (MPa) & $5^{\circ} \mathrm{PI}(\mathrm{MPa})$ & FM (\%) \\
\hline PVA-160/0,50 & $9,23 \mathrm{f}(16,92)$ & 7,64 & 2 & 2,17 b $(34,25)$ & 1,33 & 0 \\
\hline EPI-160/0,50 & $12,13 \mathrm{ab}(15,14)$ & 9,52 & 43 & 4,77 a $(15,00)$ & 3,80 & 13 \\
\hline PVA-180/0,50 & 9,64 def $(19,12)$ & 6,46 & 2 & 1,82 b $(30,88)$ & 1,24 & 0 \\
\hline EPI-180/0,50 & 11,50 abcde $(15,07)$ & 8,82 & 32 & 4,70 a $(17,39)$ & 3,43 & 3 \\
\hline PVA-200/0,50 & 10,18 bcdef $(15,10)$ & 8,07 & 8 & $1,74 \mathrm{~b}(42,60)$ & 0,85 & 1 \\
\hline EPI-200/0,50 & 11,99 abc $(11,79)$ & 10,15 & 45 & 4,97 a $(15,38)$ & 3,92 & 5 \\
\hline PVA-160/0,75 & 11,42 abcde $(10,45)$ & 9,21 & 8 & 1,95 b $(27,58)$ & 1,13 & 2 \\
\hline EPI-160/0,75 & 11,72 abc $(9,71)$ & 9,74 & 78 & 5,17 a $(12,08)$ & 4,37 & 11 \\
\hline PVA-180/0,75 & 10,55 bcdef $(17,60)$ & 7,26 & 9 & $2,09 \mathrm{~b}(31,60)$ & 1,11 & 0 \\
\hline EPI-180/0,75 & 12,66 a $(14,92)$ & 10,22 & 46 & 5,23 a $(15,47)$ & 4,15 & 18 \\
\hline PVA-200/0,75 & $9,10 f(22,94)$ & 5,98 & 10 & $2,11 \mathrm{~b}(27,67)$ & 1,17 & 1 \\
\hline EPI-200/0,75 & 10,98 abcdef $(11,77)$ & 9,04 & 56 & 4,96 a $(12,68)$ & 4,21 & 13 \\
\hline PVA-160/1,0 & $8,99 f(13,91)$ & 6,88 & 5 & $1,80 \mathrm{~b}(17,32)$ & 1,38 & 1 \\
\hline EPI-160/1,0 & 11,22 abcdef $(20,39)$ & 8,66 & 19 & 4,71 a $(19,58)$ & 3,03 & 3 \\
\hline PVA-180/1,0 & 9,51 ef $(8,43)$ & 8,36 & 12 & 1,98 b $(22,90)$ & 1,08 & 0 \\
\hline EPI-180/1,0 & 11,58 abcd $(14,23)$ & 8,98 & 40 & 4,57 a $(12,16)$ & 3,98 & 3 \\
\hline PVA-200/1,0 & 10,11 bcdef $(20,67)$ & 7,06 & 13 & $2,09 \mathrm{~b}(27,02)$ & 1,32 & 1 \\
\hline EPI-200/1,0 & 10,00 cdef $(13,83)$ & 8,24 & 36 & 4,43 a $(21,10)$ & 2,90 & 8 \\
\hline
\end{tabular}

EPI: Emulsão Polimérica de Isocianato; PVA: Poliacetato de vinila; RLC: Resistência da Linha de Cola ao Cisalhamento; $5^{\circ}$ PI: $5^{\circ}$ Percentil Inferior; FM: falhas na madeira; Médias seguidas de mesma letra, na mesma coluna, são estatisticamente iguais pelo Teste de Tukey a 95\% de confiabilidade; Valores entre parênteses indicam o coeficiente de variação.

Os valores médios de cisalhamento para pré-tratamento a seco variaram de $8,99 \mathrm{MPa}$ para juntas coladas com adesivo PVA, gramatura de $160 \mathrm{~g} / \mathrm{m}^{2}$ e pressão de $1,0 \mathrm{MPa}$ (PVA/160-1,0) a 12,66 MPa para juntas coladas com adesivo EPI, gramatura de $180 \mathrm{~g} / \mathrm{m}^{2} \mathrm{e}$ pressão de 0,75 $\mathrm{MPa}(\mathrm{EPI} / 180-0,75)$. Foram constatadas diferenças estatisticamente significativas entre os tratamentos.

Os resultados obtidos foram satisfatórios em comparação aos apresentados na literatura para madeiras de eucalipto, pinus e algumas espécies de madeiras tropicais. Para juntas coladas com adesivo PVA, Iwakiri et al. (2013) encontraram para madeiras de Eucalyptus benthamii, valor médio de cisalhamento de 9,04 MPa; Endo et al. (2017), obtiveram para madeiras Pinus taeda, valores entre 5,12 e 5,55 MPa; Bila et al. (2016) encontraram para cinco 
espécies de madeiras tropicais (Eschweilera coriacea, Manilkara amazônica, Protium puncticulatum, Inga paraensis e Byrsonima crispa) valores entre 2,24 e 8,45 MPa. Para juntas coladas com adesivo EPI, Fonte (2016) obteve para Cryptomeria japônica valor médio de 5,1 MPa; Almeida (2015) obteve para Cupressus lusitânica valor médio de 4,88 MPa; Bila et al. (2016) obteve para seis espécies de madeiras tropicais valores médios entre 7,66 a 13,76 MPa; e, Campelo et al. (2017) encontraram para Genipa americana valor médio de 4,20 MPa.

As variações observadas no $5^{\circ}$ percentil inferior foi de 5,98 MPa para juntas coladas com adesivo PVA, gramatura de $200 \mathrm{~g} / \mathrm{m}^{2}$ e pressão de $0,75 \mathrm{MPa}$ (PVA/200-0,75) a 10,22 MPa para juntas coladas com adesivo EPI, gramatura de $180 \mathrm{~g} / \mathrm{m}^{2}$ e pressão de $0,75 \mathrm{MPa}(\mathrm{EPI} / 180-0,75)$. Todos os tratamentos atenderam ao requisito mínimo de 2,5 MPa estabelecido pela norma EN 13353-2008.

Com relação à percentagem de falhas na madeira, os valores médios variaram de $2 \%$ para juntas coladas com adesivo PVA, gramatura de $160 \mathrm{~g} / \mathrm{m}^{2}$ e pressão de $0,50 \mathrm{MPa}$ (PVA/160-0,50) a 78\% para juntas coladas com adesivo EPI, gramatura de $160 \mathrm{~g} / \mathrm{m}^{2}$ e pressão de $0,75 \mathrm{MPa}$ (EPI/160-0,75). A maioria dos tratamentos com adesivo EPI atenderam ao requisito mínimo de $40 \%$ estabelecido pela norma EN 13353-2008. Por outro lado, nenhum dos tratamentos com adesivo PVA atendeu a este requisito referente às falhas na madeira, indicando uma baixa qualidade de colagem da madeira com este adesivo.

Os valores médios de cisalhamento após pré-tratamento a úmido variaram de 1,80 MPa para juntas coladas com adesivo PVA, gramatura de $160 \mathrm{~g} / \mathrm{m}^{2}$ e pressão de $1,0 \mathrm{MPa}$ (PVA/160-1,0) a 5,23 MPa para juntas coladas com adesivo EPI, gramatura de $180 \mathrm{~g} / \mathrm{m}^{2} \mathrm{e}$ pressão de 0,75 $\mathrm{MPa}(\mathrm{EPI} / 180-0,75)$. Foram constatadas diferenças estatisticamente significativas entre os tratamentos. Nas comparações com o pré-tratamento a seco, os resultados indicaram efeitos de significativos da imersão em água fria para degradação da linha de cola, com a consequente redução dos valores de cisalhamento.

Os resultados obtidos foram semelhantes aos encontrados na literatura para madeiras de pinus e algumas espécies de madeiras tropicais. Para juntas coladas com adesivo PVA, Endo et al. (2017) encontraram para Pinus taeda valores de 3,36 MPa; Iwakiri et al. (2016) obtiveram para madeiras de Protium puncticulatum e Dinizia excelsa, valores de 5,01 e $5,07 \mathrm{MPa}$, respectivamente. Para juntas coladas com adesivo EPI, Fonte \& Trianoski (2015) obteve para Cryptomeria japônica valor médio de 3,90 MPa; Lau (2017) obteve para Populus deltoides valor médio de 2,61 MPa.

As variações observadas no $5^{\circ}$ percentil inferior foi de $0,85 \mathrm{MPa}$ para juntas coladas com adesivo PVA, gramatura de $200 \mathrm{~g} / \mathrm{m}^{2}$ e pressão de $0,50 \mathrm{MPa}$ (PVA/200-0,50) a 4,37 MPa para juntas coladas com adesivo EPI, gramatura de $160 \mathrm{~g} / \mathrm{m}^{2}$ e pressão de $0,75 \mathrm{MPa}$ (EPI/160-0,75). Todos os tratamentos com adesivo EPI atenderam ao requisito mínimo de 2,5 MPa estabelecido pela norma EN 13353 (European Committee for Standardization, 2008a), indicando a maior resistência à umidade do EPI em relação ao PVA, tendo em vista que nenhum tratamento com adesivo PVA atingiu o requisito estabelecido pela referida norma.

O maior valor médio de percentagem de falhas na madeira foi de $18 \%$, para juntas coladas com adesivo EPI, gramatura de $180 \mathrm{~g} / \mathrm{m}^{2}$ e pressão de $0,75 \mathrm{MPa}$, ficando abaixo dos 40\% estabelecido pela norma EN 13353 (European Committee for Standardization, 2008a), indicando a influência do pré-tratamento com água fria no descolamento da linha de cola. Estudos realizados por Iwakiri et al. (2015a) também revelaram baixos percentuais de falhas na madeira para Inga alba e Swartzia recurva, com valores de 2,0 e 3,0\%, respectivamente, para o teste úmido, evidenciando a influência da umidade no cisalhamento da linha de cola.

\section{Efeitos do tipo de adesivo, gramatura e pressão na resistência ao cisalhamento das juntas coladas.}

Na Tabela 5 estão apresentados os resultados dos efeitos do tipo de adesivo sobre a resistência ao cisalhamento das juntas coladas.

Tabela 5. Efeitos do adesivo sobre a resistência da linha de cola ao cisalhamento 


\begin{tabular}{|c|c|c|c|c|c|c|}
\hline \multirow[b]{2}{*}{ Adesivo } & \multicolumn{3}{|c|}{ Seco (Curado) } & \multicolumn{3}{|c|}{ 24h Água fria (Úmido) } \\
\hline & RLC (MPa) & $5^{\circ} \mathrm{PI}(\mathrm{MPa})$ & $\begin{array}{l}\text { FM } \\
\text { (\%) }\end{array}$ & RLC (MPa) & $5^{\circ} \mathrm{PI}(\mathrm{MPa})$ & FM (\%) \\
\hline PVA & $8,74 \mathrm{~b}(15,06)$ & 7,02 & 44 & 8,27 a $(29,70)$ & 1,12 & 1 \\
\hline EPI & $\begin{array}{l}10,47 \mathrm{a} \\
(17,77)\end{array}$ & 8,96 & 7 & $\begin{array}{l}10,84 \mathrm{a} \\
(16,10)\end{array}$ & 3,40 & 8 \\
\hline
\end{tabular}

EPI: Emulsão Polimérica de Isocianato; PVA: Poliacetato de vinila; RLC: Resistência da Linha de Cola ao Cisalhamento; $5^{\circ} \mathrm{PI}: 5^{\circ}$ Percentil Inferior; FM: falhas na madeira; Médias seguidas de mesma letra, na mesma coluna, são estatisticamente iguais pelo Teste de Tukey a 95\% de confiabilidade; Valores entre parênteses indicam o coeficiente de variação.

Para o pré-tratamento a seco, as juntas coladas com adesivo EPI apresentaram valor médio de cisalhamento estatisticamente superior em comparação às juntas coladas com adesivo PVA. Já para o pré-tratamento a úmido, não foram constatadas diferenças significativas entre os adesivos PVA e EPI, embora este tenha apresentado valor superior em termos de média absoluta. Todos os tratamentos apesentaram baixos percentuais de falhas na madeira, não atingindo o valor mínimo de 40\%, conforme recomendado pela norma EN 13353 (European Committee for Standardization, 2008a).

Com relação ao $5^{\circ}$ percentil inferior, as juntas coladas com adesivo EPI atenderam ao requisito da norma EN 13353 (European Committee for Standardization, 2008a), tanto para o pré-tratamento a seco, quanto para o úmido, demonstrando melhor eficiência de colagem em comparação ao adesivo PVA.

Estudos realizados sobre juntas coladas de madeiras de várias espécies, indicam que o adesivo EPI apresenta melhor resistência ao cisalhamento da linha de cola em relação ao adesivo PVA, principalmente no ensaio a úmido (Fonte, 2016; Bila et al., 2016; Almeida, 2015; Campelo et al., 2017).

Na Tabela 6 estão apresentados os resultados dos efeitos da gramatura sobre a resistência ao cisalhamento das juntas coladas.

Tabela 6. Efeitos da gramatura sobre a resistência da linha de cola ao cisalhamento

\begin{tabular}{|c|c|c|c|c|c|c|}
\hline \multirow{2}{*}{$\begin{array}{l}\text { Gramatura } \\
\left(\mathrm{g} / \mathrm{m}^{2}\right)\end{array}$} & \multicolumn{3}{|c|}{ Seco (Curado) } & \multicolumn{3}{|c|}{ 24h Água fria (Úmido) } \\
\hline & RLC (MPa) & $5^{\circ} \mathrm{PI}(\mathrm{MPa})$ & FM (\%) & RLC (MPa) & $5^{\circ} \mathrm{PI}(\mathrm{MPa})$ & FM (\%) \\
\hline 160 & 7,22 c $(17,34)$ & 8,05 & 26 & 7,46 c $(45,60)$ & 1,32 & 5 \\
\hline 180 & 9,49 b $(18,14)$ & 7,51 & 23 & 9,81 b $(44,92)$ & 1,24 & 4 \\
\hline 200 & 11,83 a $(17,71)$ & 7,31 & 27 & $\begin{array}{l}11,70 \text { a } \\
(44,74)\end{array}$ & 1,17 & 5 \\
\hline
\end{tabular}

RLC: Resistência da Linha de Cola ao Cisalhamento; $5^{\circ}$ PI: $5^{\circ}$ Percentil Inferior; FM: falhas na madeira; Médias seguidas de mesma letra, na mesma coluna, são estatisticamente iguais pelo Teste de Tukey a 95\% de confiabilidade; Valores entre parênteses indicam o coeficiente de variação.

Tanto para o pré-tratamento a seco, quanto para o úmido, o aumento na gramatura resultou em maiores valores médios de resistência ao cisalhamento das juntas coladas. As percentagens de falhas na madeira foram baixas para ambos os pré-tratamentos. $O$ aumento na resistência ao cisalhamento das juntas coladas para maiores gramaturas de adesivos foi constatado também por Iwakiri et al. (2015b) para madeiras de Cryptomeria japônica e Sequoia sempervirens. Os melhores resultados de cisalhamento foram obtidos para gramatura de $200 \mathrm{~g} / \mathrm{m}^{2}$, a qual está dentro da faixa de gramaturas empregada pelas indústrias, que são de 180 a $200 \mathrm{~g} / \mathrm{m}^{2}$ para o PVA e 150 a $300 \mathrm{~g} / \mathrm{m}^{2}$ para EPI (Iwakiri et al., 2014).

Com relação ao $5^{\circ}$ percentil inferior, para todas as gramaturas os valores médios de cisalhamento obtidos para o pré-tratamento a seco foram superiores ao mínimo de 2,5 $\mathrm{MPa}$ exigido pela norma EN 13353 (European Committee for Standardization, 2008a). Já para o prétratamento a úmido, os requisitos da referida norma não foram atendidos por nenhuma das 
gramaturas de 160, 180 e $200 \mathrm{~g} / \mathrm{m}^{2}$, testadas neste estudo. As variações constatadas entre as gramaturas, indicam que o aumento na gramatura acima do valor máximo testado, não deverá melhorar os resultados de $5^{\circ}$ percentil inferior.

Na Tabela 7 estão apresentados os resultados dos efeitos da pressão sobre a resistência ao cisalhamento das juntas coladas.

Tabela 7. Efeitos da pressão sobre a resistência da linha de cola ao cisalhamento

\begin{tabular}{|c|c|c|c|c|c|c|}
\hline \multirow{2}{*}{$\begin{array}{c}\text { Pressão } \\
\text { (MPa) }\end{array}$} & \multicolumn{3}{|c|}{ Seco (Curado) } & \multicolumn{3}{|c|}{ 24h Água fria (Úmido) } \\
\hline & RLC (MPa) & $5^{\circ} \mathrm{PI}(\mathrm{MPa})$ & FM (\%) & RLC (MPa) & $5^{\circ} \mathrm{PI}(\mathrm{MPa})$ & FM (\%) \\
\hline 0,50 & $3,59 \mathrm{c}(18,25)$ & 7,53 & 22 & 3,58 c $(46,12)$ & 1,23 & 4 \\
\hline 0,75 & $9,37 \mathrm{~b}(17,00)$ & 7,57 & 35 & 9,42 b $(45,30)$ & 1,17 & 8 \\
\hline 1,0 & $\begin{array}{l}15,83 \mathrm{a} \\
(17,57)\end{array}$ & 7,39 & 21 & 15,68 a $(43,25)$ & 1,37 & 3 \\
\hline
\end{tabular}

RLC: Resistência da Linha de Cola ao Cisalhamento; $5^{\circ}$ PI: $5^{\circ}$ Percentil Inferior; FM: falhas na madeira; Médias seguidas de mesma letra, na mesma coluna, são estatisticamente iguais pelo Teste de Tukey a 95\% de confiabilidade; Valores entre parênteses indicam o coeficiente de variação.

O aumento na pressão de prensagem de 0,50 para 0,75 e 1,0 MPa, resultaram em valores médios de cisalhamento estatisticamente superiores, tanto para o pré-tratamento a seco, quanto para o úmido. Segundo a literatura, há um nível adequado de pressão de colagem para madeiras de acordo com a sua massa específica. Marra (1992) afirma que quanto maior a massa específica da madeira maiores níveis de pressão são requeridos, e, neste estudo, os resultados indicam que a pressão de 1,0 MPa foi a mais indicada para a colagem da madeira de E. badjensis. As percentagens de falhas na madeira foram inferiores a $40 \%$ para os três níveis de pressão avaliados, para os dois pré-tratamentos.

Com relação ao $5^{\circ}$ percentil inferior, para todos os níveis de pressão os valores médios de cisalhamento obtidos para o pré-tratamento a seco foram superiores ao mínimo de 2,5 MPa exigido pela norma EN 13353 (European Committee for Standardization, 2008a). Já para o pré-tratamento a úmido, os requisitos da referida norma não foram atendidos para os três níveis de pressão avaliados.

\section{CONCLUSÕES}

A madeira de Eucalyptus badjensis apresenta média massa específica básica e alta anisotropia de contração.

Os resultados de resistência à flexão estática e tração paralela das emendas de topo atenderam aos requisitos mínimos da norma ASTM D 5572, para os pré-tratamentos a seco e ciclo triplo.

Os resultados de cisalhamento da linha de cola foram superiores para as juntas coladas com adesivo EPI e para maiores gramaturas e pressão específica.

Com base nos requisitos da norma EN 15353-2008 para $5^{\circ}$ percentil inferior, pode se afirmar que a madeira de Eucalyptus badjensis apresenta grande potencial para produção de painéis EGP, com adesivo EPI, gramatura de $200 \mathrm{~g} / \mathrm{m}^{2}$ e pressão específica de 1,0 $\mathrm{MPa}$.

\section{REFERÊNCIAS}

Akzo Nobel Perú S. A. C. (2010). Información del producto EPI 1911 con HARDENER 1999. Casco Adhesives (Vol. 2). Medellín.

Almeida, C. C. F. (2015). Avaliação da qualidade da colagem da madeira de Cupressus lusitanica Mill. para a produção de painéis colados lateralmente (EDGE GLUED PANEL - EGP). $142 \mathrm{f}$. Dissertação (Mestrado em Engenharia Florestal) - Centro de Ciências Agroveterinárias, Universidade do Estado de Santa Catarina, Lages.

American Society for Testing and Materials - ASTM. (2005). ASTM. 5572: Standard specification for adhesives used for finger joints in nonstructural lumber products. West Conshohocken: ASTM. 
Arostegui, A. (1982). Recopilación y análisis de estudios tecnológicos de maderas peruanas (Documento de trabajo $n^{\circ} 2$, Proyecto PNUD/FAO/PER/81/002). Lima.

Bila, N. F., Iwakiri, S., Trianoski, R., \& Prata, J. G. (2016). Avaliação da qualidade de juntas coladas de seis espécies de madeiras tropicais da Amazônia. Floresta, 46(4), 455-464.

Campelo, S., Iwakiri, S., Trianoski, R., \& Agiar, O. R. (2017). Utilização da madeira de Genipa americana para produção de paineis de coalgem lateral EGP. Floresta, 47(1), 129-135. http://dx.doi.org/10.5380/rf.v47i1.48453.

Comissão Panamericana de Normas Técnicas - COPANT. (1972a). COPANT 459 - maderas: determinación del retratibilidad. La Paz, 1972.

Comissão Panamericana de Normas Técnicas - COPANT. (1972b). COPANT 461 - maderas: determinación del peso específico aparente. La Paz.

European Committee for Standardization - CEN. (2008a). European Standard EN 13353 - Solid wood panels (SWP) - Requirements. Bruxelas: CEN.

European Committee for Standardization - CEN. (2008b). European Standard EN 13354 - Solid wood panels (SWP) - bonding quality. Bruxelas: CEN.

Endo, C., Trianoski, R., \& Iwakiri, S. (2017). Produção de EGP com diferentes adesivos PVAc e sistemas de prensagem. Floresta e Ambiente, 24(0), 1-8. http://dx.doi.org/10.1590/2179-8087.147415.

Fonte, A. P. N., \& Trianoski, R. (2015). Efeito da gramatura sobre a qualidade de colagem lateral da madeira de Tectona grandis. Revista de Ciências Agroveterinárias, 4(3), 224-233. http://dx.doi.org/10.5965/223811711432015224.

Fonte, A. P. N. (2016). Utilização da madeira de Cryptomeria japonica para a produção de painéis colados lateralmente e aplicação de acabamento superficial. 185 f. (Dissertação de mestrado). Setor de Ciências Agrárias, Universidade Federal do Paraná, Curitiba.

Frihart, C. R., \& Hunt, C. G. (2010). Adhesives with wood materials: bond formation and performance. In: Forest Products Laboratory. Wood Handbook - Wood as an engineering material. Madison: USDA.

Higa, R. C. V., Higa, A. R., \& Alves, E. C. Comportamento de progênies de Eucalyptus badjensis Beuzev. \& Welch em dois locais da Região Sul do Brasil. Boletim de Pesquisa Florestal, 45, 2002.

Iwakiri, S. (2005). Painéis de madeira reconstituída (254 p.). Curitiba: FUPEF.

Iwakiri, S., Trianoski, R., Cunha, A. B., Prata, J. G., Hara, M., Bila, N. F., Luis, R. C. G., Araujo, R. D., \& Villas Bôas, B. T. (2013). Avaliação da resistência de juntas coladas da madeira de Eucalyptus benthamii com diferentes adesivos e faces de colagem. Scientia Forestalis, 41(99), 411-416.

Iwakiri, S., Matos, J. L. M., Prata, J. G., Trianoski, R., Parchen, C. F., Castro, V. G., \& Iwakiri, V. T.. (2014). Características de vigas laminadas coladas confeccionadas com madeira de teca (Tectona grandis). Floresta e Ambiente, 21(2), 269-275. http://dx.doi.org/10.4322/floram.2014.036.

Iwakiri, S., Trianoski, R., Nascimento, C. C., Gumane, C., Lengowski, E. C., Schardosin, F. A., \& Azambuja, R. (2015a). Resistência das juntas coladas de madeiras de Inga alba (SW) Wild e Swartzia recurva Poep. Cerne, 21(3), 457-463. http://dx.doi.org/10.1590/01047760201521031844.

Iwakiri, S., Trianoski, R., França, R. F., Gonçalves, T. A. P., Loiola, P. L., Campelo, S. R., \& Farias, R. F. (2015b). Avaliação da resistência de juntas coladas da madeira de Cryptomeria japônica e Sequoia sempervirens com diferentes adesivos. Scientia Forestalis, 43(105), 19-26.

Iwakiri, S., Trianoski, R., Fonte, A. P. N., França, M. C., Lau, P. C., \& Molleken, R. (2016). Potencial de uso de madeiras de Dinizia Ducke e Protium puncticulatum J. F. Mach para produção de pain excelsa éis EGP. Scientia Forestalis, 44(111), 709-717.

Juízo, C. G. F. (2015). Aproveitamento de costaneiras em serraria de eucalipto para produção de painéis EGP. 101 f. Dissertação (Mestrado em Engenharia Florestal) - Setor de Ciências Agrárias, Universidade Federal do Paraná, Curitiba,.

Keinert Junior, S., Rozas, E. C. E., Esturion, J. A., Matsunaga, D. K., Michaque, M. A. M., \& Rincoski, C. R. (1992). Relação entre a contração e o teor de umidade da madeira de Pinus taeda e Pinus elliottii, em vários ângulos de grã. Ciência Florestal, 2(1), 81-86.

Klitzke, R. J. (2007). Secagem da madeira - Tecnologias aplicadas ao setor florestal brasileiro (pp. 271-341). Jerônimo Monteiro: Suprema Gráfica e Editora.

Lau, P. M. (2017). Produção de paineis de colagem lateral - EGP com madeira de Populus deltoides $100 \mathrm{p}$ (Dissertação de Mestrado). Setor de Ciências Agrárias, Universidade Federal do Paraná, Curitiba.

Lobão, M. S., Castro, V. R., Rangel, A., Sarto, C., Tomazello Filho, M., Silva Junior, F. G., Camargo Neto, L., \& Bermudez, M. A. R. C. (2011). Agrupamento de espécies florestais por análises univariadas e 
multivariadas das características anatômica, física e química das suas madeiras. Scientia Forestalis, $18(1), 16-29$.

Lopes, M. C. (2008). Espectroscopia no infravermelho próximo aplicada a classificação de painéis colados lateralmente de madeira de Pinus taeda L 140 f. (Tese de Doutorado). Setor de Ciências Agrárias, Universidade Federal do Paraná, Curitiba.

Lorenzi, H. (2002). Árvores brasileiras: manual de identificação e cultivo de plantas arbóreas nativas do Brasil. Nova Odessa: Instituto Plantarum.

Mantero, C., Fernández, V., \& Roz, A. (2008). Anatomía, peso específico aparente de la madera y porcentaje de corteza en orígenes de dos especies de Eucalyptus cultivadas em Uruguay (Eucalyptus dunnii Maiden y Eucalyptus badjensis Beuzev. \& Welch). Agrociencia, 22(1), 20-30.

Marra, A. A. (1992). Technology of wood bonding: Principles in practice (453 p.). New York: Van Nostrand Reinhold.

Pizzi, A. (1983). Wood adhesives: chemistry and technology (364 p.). New York: Marcel Dekker.

Prata, J. G. (2010). Estudo da viabilidade tecnológica do uso de espécies de pinus tropicais para produção de painéis colados lateralmente (Edge Glued Panels - EGP). (Tese de Doutorado). Setor de Ciências Agrárias, Universidade Federal do Paraná, Curitiba.

Serrano, L. J. P. Resistência à tração da madeira e resistência ao cisalhamento no plano de cola, seus impactos na geometria do dente e propriedades mecânicas de emendas dentadas. 107 f. (Dissertação de Mestrado). Universidade de São Paulo, Piracicaba, 2009. http://dx.doi.org/10.11606/D.11.2009.tde-14042009-111712.

Tienne, D. L. C. Qualidade da adesão de juntas coladas expostas a condições de serviço externo e interno. 68 f. (Dissertação de Metrado). Instituto de Florestas, Universidade Federal Rural do Rio de Janeiro, Seropédica, 2006.

Tienne, D. L. C., Nascimento, A. M., Garcia, R. A., \& Silva, D. B. (2011). Qualidade de adesão de juntas de madeira de Pinus coladas em condições simuladas de serviço interna e externa. Floresta e Ambiente, 18(1), 16-29. http://dx.doi.org/10.4322/floram.2011.019.

Tsoumis, G. Science and technology of wood: structure, properties, utilization. New York: Chapmam \& Hall, 1991, $494 \mathrm{p}$.

Vick, C. B. (1999). Adhesive bonding of wood materials. In United States Department of Agriculture. Wood handbook - Wood as an engineering material. Madison: U.S. Products Laboratory, Department of Agriculture, Forest Service, Forest Products Laboratory.

Contribuição dos Autores: SI: Conceituação, análise formal, primeira redação, revisão e edição; RT: Conceituação, curadoria de dados, análise formal, investigação, metodologia, supervisão, validação; RRZ: curadoria de dados, investigação, metodologia, validação; GFP: curadoria de dados, investigação, metodologia, validação; TSR: curadoria de dados, investigação, metodologia, 